\title{
On the sum of three arbitrary Fibonacci and Lucas numbers
}

\author{
Nurettin Irmak ${ }^{1}$, Zafer Şiar $^{2}$ and Refik Keskin ${ }^{3}$ \\ ${ }^{1}$ Department of Mathematics, Ömer Halisdemir University \\ Niğde, Turkey \\ e-mail: nirmak@ohu.edu.tr \\ ${ }^{2}$ Department of Mathematics, Bingöl University \\ Bingöl, Turkey \\ e-mail: zsiar@bingol.edu.tr \\ ${ }^{3}$ Department of Mathematics, Sakarya University \\ Sakarya, Turkey \\ e-mail: rkeskin@sakarya.edu.tr
}

Received: 9 January 2019

Abstract: In this paper, we solve the equations

$$
\begin{aligned}
& L_{k}=F_{n}+F_{m}+F_{r}, \\
& F_{k}=F_{n}+F_{m}+F_{r}, \\
& L_{k}=L_{n}+L_{m}+L_{r}, \\
& F_{k}=L_{n}+L_{m}+L_{r}
\end{aligned}
$$

for $0<r \leq m \leq n$ and a natural number $k$. It is shown that only the equation $F_{k}=L_{n}+L_{m}+L_{r}$ has a finite number of solutions. The others have infinitely many solutions.

Keywords: Fibonacci number, Lucas number, Recurrences.

2010 Mathematics Subject Classification: 11B39, 11D61, 11 B37.

\section{Introduction}

The Fibonacci sequence $\left(F_{n}\right)$ is defined as $F_{0}=0, F_{1}=1$, and $F_{n}=F_{n-1}+F_{n-2}$ for $n \geq 2$. The Lucas sequence $\left(L_{n}\right)$, which is similar to the Fibonacci sequence, is defined by the same 
recursive pattern with initial conditions $L_{0}=2, L_{1}=1 . F_{n}$ and $L_{n}$ are called the $n$-th Fibonacci number and the $n$-th Lucas number, respectively. These two sequences are the most important among the second order linear recursive sequences and have been investigated by the researchers. Firstly, square terms and later perfect powers in the Fibonacci and Lucas sequences have attracted the attention of the researchers. The problem of finding all perfect powers in these sequences had remained an open problem for many years. It was solved in 2006 by Bugeaud, Mignotte and Siksek in [1]. It is shown that the perfect powers in the Fibonacci and Lucas sequences are $F_{0}=0, F_{1}=F_{2}=1, F_{6}=8=2^{3}, F_{12}=144=12^{2}$, and $L_{1}=1, L_{3}=4=2^{2}$, respectively. In [4], the authors showed that the equation $L_{r}=L_{m} L_{n}$ is impossible for $m>1$ and $n>1$. In [3], Farrokhi proved that if $m>2$ and $n>2$, then there is no Fibonacci number $F_{n}$ such that $F_{r}=F_{m} F_{n}$. Similar equations were tackled by Carlitz in [2]. It is natural to ask when the sum of three Fibonacci numbers is a Lucas number or a Fibonacci number? And when the sum of three Lucas numbers is a Fibonacci number or a Lucas number?

In this paper, we tackle these problems and we solve the equations

$$
\begin{aligned}
& L_{k}=F_{n}+F_{m}+F_{r} \\
& F_{k}=F_{n}+F_{m}+F_{r}, \\
& F_{k}=L_{n}+L_{m}+L_{r}, \\
& L_{k}=L_{n}+L_{m}+L_{r},
\end{aligned}
$$

and find all solutions $n, m, r$ and $k$ in positive integers. It is seen that only the equation (3) has a finite number of solutions. The others have infinitely many solutions.

There are many amazing identities between Fibonacci and Lucas numbers. One of them, which will be used later, is

$$
F_{n+1}+F_{n-1}=L_{n}
$$

It is well known that

$$
\alpha^{n-2} \leq F_{n} \leq \alpha^{n-1}
$$

and

$$
\alpha^{n-1} \leq L_{n} \leq 2 \alpha^{n}
$$

where $\alpha=(1+\sqrt{5}) / 2$ is the Golden section.

\section{Auxiliary results}

The following lemma can be concluded from Lemma 7 and Corollary 8 of [6]. This lemma gives more precise upper and lower bounds for the Fibonacci and Lucas numbers.

Lemma 2.1. For all integers $n \geq 8$, the two inequalities

$$
\alpha^{n-0.01}<L_{n}<\alpha^{n+0.01}
$$


and

$$
\alpha^{n-1.68}<F_{n}<\alpha^{n-1.67}
$$

hold.

We can write the next lemma from Theorem 8.1 and Corollary 8.1 given in [5].

Lemma 2.2. Let $n \geq 1$ be an integer. Then

$$
F_{n}=\frac{\alpha^{n}}{\sqrt{5}}+e_{n}
$$

with $\left|e_{n}\right|<1 / 2$.

The solutions of the equation $F_{n}+F_{m}=F_{r}$ for $1<m<n$ can be found in [2]. For the sake of completeness, we solve this equation for $1 \leq m \leq n$.

Lemma 2.3. Let $1 \leq m \leq n$. Then all solutions of the equation $F_{n}+F_{m}=F_{r}$ are the elements of the set

$$
(n, m, r) \in\{(n, n-1, n+1),(1,1,3),(2,2,3),(3,1,4)\} .
$$

Proof. Let $1 \leq m \leq n$. If $n \leq 3$, one can easily see that $(n, m, r)=(1,1,3),(2,1,3)$, $(2,2,3),(3,1,4)$ and $(3,2,4)$. Assume that $n \geq 4$. From (6), we get

$$
\alpha^{r-1} \geq F_{r}=F_{m}+F_{n}>F_{n} \geq \alpha^{n-2},
$$

which implies that $n-1<r$. Also, since

$$
\alpha^{r-2} \leq F_{r}=F_{m}+F_{n} \leq \alpha^{m-1}+\alpha^{n-1} \leq 2 \alpha^{n-1}<\alpha^{2} \alpha^{n-1}=\alpha^{n+1},
$$

it follows that $r<n+3$. Consequently, $n-1<r<n+3$. Now, we separate three cases into the proof:

Case 1. If $r=n$, then we get $F_{m}=0$, which contradicts the fact that $m \geq 1$.

Case 2. If $r=n+2$, then we get $F_{n+1}=F_{m}$. Since $n+1 \geq 5$, this is possible only for $n+1=m$, which contradicts the fact that $m \leq n$.

Case 3. If $r=n+1$, then we get $F_{n+1}=F_{r}=F_{m}+F_{n}$ and thus $F_{m}=F_{n-1}$. Since $n-1 \geq 3$, it follows that $m=n-1$. Hence, $(n, m, r)=(n, n-1, n+1)$ is a solution of the equation $F_{n}+F_{m}=F_{r}$. This completes the proof.

The proofs of the following results can be done similarly.

Lemma 2.4. Let $1 \leq m \leq n$. Then the equation $F_{n}+F_{m}=L_{r}$ has only the solutions $(n, m, r)=$ $(n, n-2, n-1),(3,2,2),(3,3,3)$, and $(4,1,3)$.

Lemma 2.5. Let $1 \leq m \leq n$. Then the equation $L_{n}+L_{m}=F_{r}$ has only the solutions $(n, m, r)=$ $(1,1,3),(3,1,5),(4,1,6),(6,2,8)$, and $(3,3,6)$.

Lemma 2.6. Let $1 \leq m \leq n$. Then the equation $L_{n}+L_{m}=L_{r}$ has only the solution $(n, m, r)=$ $(n, n-1, n+1)$. 


\section{Main theorem}

From now on, it will be assumed that $k, r, m, n$ are natural numbers and $1 \leq r \leq m \leq n$.

Theorem 3.1. All solutions of the Diophantine equation $L_{k}=F_{n}+F_{m}+F_{r}$ are given by

$$
(n, m, r, k) \in\{(n, n, n-3, n),(n, n-1, n-1, n),(n, n-3, n-4, n-1)\}
$$

or

$$
\begin{gathered}
(n, m, r, k) \in\{(1,1,1,2),(2,2,1,2),(2,2,2,2),(3,1,1,3),(3,2,1,3),(4,4,2,4),(5,1,1,4), \\
(5,2,2,4),(5,5,1,5),(6,3,1,5)\} .
\end{gathered}
$$

Proof. Assume that the equation (1) holds for $8 \leq r \leq m \leq n$. This implies that $k \leq 8$. Then, since $L_{k}=F_{n}+F_{m}+F_{r} \leq 3 F_{n}$, from Lemma 2.1, we can write

$$
\alpha^{k-0.01}<L_{k} \leq 3 F_{n} \leq 3 \alpha^{n-1.67}<\alpha^{n-1.67+2.29} .
$$

The last inequality implies that $k-n<0.63$. Also, since $F_{n} \leq L_{k}$, it follows from Lemma 2.1 that $\alpha^{n-1.68}<\alpha^{k+0.01}$, which implies that $-1.69<k-n$.

Consequently, we have $-1.69<k-n<0.63$. This shows that $k-n=-1$ or $k-n=0$. On the other hand, using Lemma 2.2, we can write

$$
\alpha^{k}+\beta^{k}=\frac{\alpha^{n}}{\sqrt{5}}+\frac{\alpha^{m}}{\sqrt{5}}+\frac{\alpha^{r}}{\sqrt{5}}+e_{n}+e_{m}+e_{r}
$$

and so

$$
\begin{aligned}
\left|\alpha^{k}-\frac{\alpha^{n}}{\sqrt{5}}\right| & =\left|\frac{\alpha^{m}}{\sqrt{5}}+\frac{\alpha^{r}}{\sqrt{5}}-\beta^{k}+e_{n}+e_{m}+e_{r}\right| \\
& \leq \frac{1}{\sqrt{5}}\left(\alpha^{m}+\alpha^{r}\right)+|\beta|^{k}+\left|e_{n}\right|+\left|e_{m}\right|+\left|e_{r}\right| \\
& \leq \frac{1}{\sqrt{5}}\left(\alpha^{m}+\alpha^{r}\right)+1.572 .
\end{aligned}
$$

Dividing the last inequality by $\alpha^{k}$, we get

$$
\left|1-\frac{\alpha^{n-k}}{\sqrt{5}}\right| \leq \frac{1}{\sqrt{5}}\left(\frac{1}{\alpha^{k-m}}+\frac{1}{\alpha^{k-r}}\right)+\frac{1.572}{\alpha^{k}} \leq\left(\frac{2}{\sqrt{5}}+1.572\right) \frac{1}{\alpha^{k-m}}<\frac{2.4665}{\alpha^{k-m}} .
$$

If $k-n=0$ or $k-n=-1$, the above inequality gives us that $k-m<3.12$ or $k-m<4.552$. That is, $k-m \leq 4$. Also, since $F_{m} \leq L_{k}$, it follows that $\alpha^{m-1.68}<\alpha^{k+0.01}$, which implies that $-1.69<k-m$. Consequently, we have $-1 \leq k-m \leq 4$. Therefore, $k-m=-1,0,1,2,3$, or 4 .

Now, we separate the proof into two cases: $k-n=0$ and $k-n=-1$.

Case 1. Let $k=n$. In this case, it is impossible that $k-m=-1$ since $n \geq m$. If $k-m=0$, then the equation (1) implies that $L_{n}=F_{n}+F_{n}+F_{r}$. Using (5), one can see easily that $F_{n-3}=F_{r}$, which yields that $r=n-3$ since $n-3 \geq 5$. That is, $(n, m, r, k)=(n, n, n-3, n)$ is a solution 
of (1). If $k-m=1$, then we have $L_{n}=F_{n}+F_{n-1}+F_{r}$, which implies that $r=n-1$ since $n-3 \geq 5$. Thus $(n, m, r, k)=(n, n-1, n-1, n)$ is a solution of (1). If $k-m=2$, then we get $F_{n-1}+F_{n-3}=F_{r}$ from the equation (1). This is impossible by Lemma 2.3. Similarly, if $k-m=3$, then we obtain $F_{n}=F_{r}$, which implies that $n=r$ since $n \geq 8$. This contradicts the fact that $r \leq m$. If $k-m=4$, then one can conclude from (1) that $F_{n-1}+2 F_{n-3}=F_{r}$. This shows that $F_{n-1}+2 F_{n-3} \leq F_{n}$ and thus $F_{n-3} \leq F_{n-4}$, which is impossible since $n \geq 8$.

Case 2. Let $k-n=-1$. It can be seen that the cases $k-m=-1,0$, and 1 are impossible. If $k-m=2$, then we obtain $F_{n-4}=F_{r}$ and so $r=n-4$. Thus, $(n, m, r, k)=(n, n-3, n-4, n-1)$ is a solution of (1). If $k-m=3$, then we obtain $F_{n-3}=F_{r}$, and so $r=n-3$. This contradicts the fact that $r \leq m$. Similarly, if $k-m=4$, then we get $2 F_{n-4}=F_{r}$. This implies that $n-4 \leq r$, which contradicts the fact that $r \leq m$.

Now assume that $0<r \leq m \leq n \leq 8$. Then since $L_{k}=F_{n}+F_{m}+F_{r} \leq 3 F_{8}=63$, it follows that $k \leq 8$. With the help of the Mathematica program, for $k \leq 8$, we obtain only the solutions

$$
\begin{aligned}
(n, m, r, k) \in\{ & (1,1,1,2),(2,1,1,2),(2,2,1,2),(2,2,2,2),(3,1,1,3),(3,2,1,3),(3,2,2,3), \\
& (4,3,3,4),(4,4,1,4),(4,4,2,4),(5,1,1,4),(5,2,1,4),(5,2,2,4),(5,4,4,5), \\
& (5,5,1,5),(5,5,2,5),(6,3,1,5),(6,3,2,5),(6,5,5,6),(6,6,3,6),(7,4,3,6), \\
& (7,6,6,7),(7,7,4,7),(8,5,4,7),(8,7,7,8),(8,8,5,8)\}
\end{aligned}
$$

in the range $0<r \leq m \leq n \leq 8$. Comparing all the solutions found in the above, we get the result.

We can give the following results without proof, since their proofs are similar to these of Theorem 3.1 and Lemma 2.3.

Theorem 3.2. The Diophantine equation $F_{k}=L_{n}+L_{m}+L_{r}$ has only the solutions

$$
\begin{gathered}
(n, m, r, k) \in\{(1,1,1,4),(2,1,1,5),(3,2,1,6),(4,2,2,7),(4,4,4,8),(5,1,1,7), \\
(5,4,2,8),(7,3,1,9),(8,3,3,10),(8,4,1,10),(10,6,2,12)\} .
\end{gathered}
$$

Theorem 3.3. All solutions of the Diophantine equation $F_{k}=F_{n}+F_{m}+F_{r}$ are given by

$$
(n, m, r, k) \in\{(n, n-2, n-3, n+1),(n, n, n-1, n+2)\}
$$

or

$(n, m, r, k) \in\{(1,1,1,4),(4,1,1,5),(4,2,2,5),(5,3,1,6),(2,1,1,4),(2,2,2,4),(3,3,1,5)\}$.

Theorem 3.4. The Diophantine equation $L_{k}=L_{n}+L_{m}+L_{r}$ has only the solutions $(n, m, r, k)=$ $(n, n-2, n-3, n+1),(n, n, n-1, n+2)$, and $(n, m, r, k)=(1,1,1,2)$. 


\section{References}

[1] Bugeaud, Y., Mignotte, M. \& Siksek, S. (2006). Classical and modular approaches to exponential Diophantine equations I. Fibonacci and Lucas perfect powers, Annals of Mathematics, 163 (3), 969-1018.

[2] Carlitz, L. (1964). A note on Fibonacci numbers, The Fibonacci Quarterly, 2 (1), 15-28.

[3] Farrokhi, D. G. M. (2007). Some remarks on the equation $F_{n}=k F_{m}$ in Fibonacci numbers, Journal of Integer Sequences, 10, Article 07.5.7.

[4] Keskin, R. \& Demirtürk Bitim, B. (2011). Fibonacci and Lucas congruences and their applications, Acta Mathematica Sinica (English Series), 27 (4), 725-736.

[5] Koshy, T. (2001). Fibonacci and Lucas Numbers with Applications, John Wiley and Sons, Proc., New York-Toronto.

[6] Luca, F., \& Szalay, L. (2009). Lucas Diophantine Triples, Integers, 9, 441-457. 